\title{
Connes amenability of $l^{1}$-Munn algebras
}

\author{
Ali Ghaffari, Samaneh Javadi and Ebrahim Tamimi
}

\begin{abstract}
In this paper, we study Connes amenability of $l^{1}$-Munn algebras. We apply this results to semigroup algebras. We show that for a weakly cancellative semigroup $S$ with finite idempotents, amenability and Connes amenability are equivalent.
\end{abstract}

Keywords. Amenability, Banach algebras, derivation, $l^{1}$-Munn algebras,semigroup algebras

\section{Introduction}

In [4], Eslamzadeh introduced $l^{1}$-Munn algebras. He used these algebras to characterize amenable semigroup algebras. A special case of these algebras was introduced by Munn [18]. $l^{1}$-Munn algebras has been studied in some texts. In [1], Blackmore showed the $l^{1}$-Munn algebra of the group algebra $l^{1}(G)$ is weakly amenable. Eslamzadeh in [5] and [6] investigated the structure of $l^{1}$-Munn algebras. Duncan and Paterson used the $l^{1}$-Munn algebras to study of semigroup algebras of completely simple semigroups [3].

The motivation to study of the theory of amenable von Neumann algebras stems from the fact that they are dual. In [12], it is shown that if $\mathcal{A}$ is a von Neumann algebra containing a weak*-dense amenable $C^{*}$-subalgebra, then for every normal Banach $\mathcal{A}$-bimodule $E$, every weak*-continuous derivation $D: \mathcal{A} \rightarrow E$ is inner. This concept of amenability was called Connes amenability [9]. In [21], Runde extended the notion of Connes-amenability to dual Banach algebras. For a locally compact group $G$, the group algebra $l^{1}(G)$ and the measure algebra $M(G)$ are two examples of dual Banach algebras. In [23], Runde introduced normal, virtual diagonals for a dual Banach algebra and showed that the existence of a normal virtual diagonal for $M(G)$ is equivalent to it being Connes amenable. Also in [22], it is shown that $G$ is amenable if and only if $M(G)$ is Connes amenable. In particular, $l^{1}(G)$ is amenable if and only if $l^{1}(G)$ is Connes amenable.

The investigation of Connes amenability for dual Banach algebras which are not von Neumann algebra is interesting for many authors, see [24], [2] and [7]. Several authors have generalized the earlier concept of amenability introduced by Lau in [13] (see [14], [15], [16] and [17]). Recently the authors have introduced the $\phi$-version of Connes amenability of dual Banach algebra

Received date: December 11, 2019; Published online: April 16, 2021.

2010 Mathematics Subject Classification. 65F05, 46L05, 11Y50.

Corresponding author: Ali Ghaffari. 
$\mathcal{A}$ that $\phi$ is a homomorphism from $\mathcal{A}$ onto $\mathbb{C}$ that lies in predual $\mathcal{A}_{*}$. We study the Runde's theorem for the case of semigroup algebra of a weakly cancellative semigroup [8]. In this paper, we study Connes amenability of $l^{1}$-Munn algebras. We use the $l^{1}$-Munn algebras to study of Connes amenability of semigroup algebras of weakly cancellative semigroups. In order to do this, we follow the argument of [4].

\section{Connes amenability of $l^{1}$-Munn algebras}

Let $\mathcal{A}$ be a dual Banach algebra with predual $\mathcal{A}_{*}$. A dual Banach $\mathcal{A}$-bimodule $E$ is called normal Banach $\mathcal{A}$-bimodule if for each $x \in E$, the maps $a \mapsto x . a, a \mapsto a . x$ are weak*-continuous $(a \in \mathcal{A})$. $\mathcal{A}$ is called Connes amenable, if for every normal Banach $\mathcal{A}$-bimodule $E$, every weak*-continuous derivation $D: \mathcal{A} \rightarrow E$ is inner.

Let $E$ be a Banach $\mathcal{A}$-bimodule. An element $x \in E$ is called weak ${ }^{*}$-weakly continuous if the module maps $a \mapsto x . a, a \mapsto a . x$ are weak*-weak continuous $(a \in \mathcal{A})$. The collection of all weak*weakly continuous elements of $E$ is denoted by $\sigma w c(E)$. It is shown that, $\sigma w c(E)^{*}$ is normal [24]. Let $\pi: \mathcal{A} \widehat{\otimes} \mathcal{A} \rightarrow \mathcal{A}$ be the multiplication map. From Corollary 4.6 in [24], $\pi^{*}$ maps $\mathcal{A}_{*}$ into $\sigma w c\left((\mathcal{A} \widehat{\otimes} \mathcal{A})^{*}\right)$. Consequently, $\pi^{* *}$ drops to a homomorphism $\pi_{\sigma w c}: \sigma w c\left((\mathcal{A} \widehat{\otimes} \mathcal{A})^{*}\right)^{*} \rightarrow \mathcal{A}$. An element $M \in \sigma w c\left((\mathcal{A} \widehat{\otimes} \mathcal{A})^{*}\right)^{*}$ is called a $\sigma w c$-virtual diagonal for $\mathcal{A}$, if $M . u=u . M, u . \pi_{\sigma w c}(M)=$ $u$ for every $u \in \mathcal{A}$. In [24], Runde showed that $\mathcal{A}$ is Connes amenable if and only if there is a $\sigma w c$-virtual diagonal for $\mathcal{A}$.

Let $\mathcal{A}$ be a unital Banach algebra, let $I$ and $J$ be nonempty sets and $P=\left(p_{i j}\right) \in M_{J \times I}(\mathcal{A})$ be such that $\|P\|_{\infty}=\sup \left\{\left\|p_{j i}\right\|: j \in J, i \in I\right\} \leq 1$. The set $M_{I \times J}(\mathcal{A})$ of all $I \times J$ matrices $a=\left(a_{i j}\right)$ on $\mathcal{A}$ with $l^{1}$-norm and the product $A \odot B=A P B,\left(A, B \in M_{I \times J}(\mathcal{A})\right)$ is a Banach algebra that is called $l^{1}$-Munn algebra on $\mathcal{A}$ with sandwich matrix $P$. It is denoted by $\mathcal{L} \mathcal{M}(\mathcal{A}, P, I, J)$ [4]. Also $\xi_{i j}$ is denoted the element of $M_{I \times J}(\mathbb{C})$ with 1 in $(i, j)$ th place and 0 elsewhere. Throughout we use the notations of [4]. We define $\Gamma: M_{I \times J}\left(\mathcal{A}_{*}\right) \rightarrow M_{I \times J}(\mathcal{A})$ by $\left\langle(\Gamma(f))_{i j}, a \xi_{i j}\right\rangle \rightarrow\left\langle\left(f_{i j}\right), a \xi_{i j}\right\rangle$, then $M_{I \times J}(\mathcal{A})$ is a dual space with predual $M_{I \times J}\left(\mathcal{A}_{*}\right)$. It is clear that above multiplication in $\mathcal{L} \mathcal{M}(\mathcal{A}, P, I, J)$ is separately weak*-continuous and from Proposition 1.2 in $[21], \mathcal{L M}(\mathcal{A}, P, I, J)$ is a dual Banach algebra.

Theorem 2.1. Let $\mathcal{A}$ be a unital dual Banach algebra. The following are equivalent:

(i) $\mathcal{L} \mathcal{M}(\mathcal{A}, P, I, J)$ is Connes amenable;

(ii) $\mathcal{A}$ is Connes amenable, $I$ and $J$ are finite and $P$ is invertible.

Proof. (i) $\Rightarrow$ (ii) Since $\mathcal{L} \mathcal{M}(\mathcal{A}, P, I, J)$ is Connes amenable dual Banach algebra, then from Proposition 4.1 in [21], it has a bounded approximate identity. By Lemma 3.7 and Lemma 3.5 in [4], $I$ and $J$ are finite, $P$ is invertible and $\mathcal{L M}(\mathcal{A}, P, I, J)$ is topologically algebra isomorphic to $\mathcal{L} \mathcal{M}\left(\mathcal{A}, I_{m}, I, J\right)$ where $I_{m}$ is the identity matrix with dimension $m$ and $|I|=|J|=m$. It is known that $\mathcal{L} \mathcal{M}\left(\mathcal{A}, I_{m}, I, J\right)$ is isometrically algebra isomorphic to $\mathcal{M}_{m} \widehat{\otimes} \mathcal{A}$ where $\mathcal{M}_{m}$ is the algebra of $m \times m$ complex matrices [19]. Using the idea of Theorem 4.1 in [4] and Theorem 4.8 in [24], we obtain the desired proof.

By Theorem 4.8 in [24], there exists

$$
M \in \sigma w c\left(\left(\left(\mathcal{M}_{m} \widehat{\otimes} \mathcal{A}\right) \widehat{\otimes}\left(\mathcal{M}_{m} \widehat{\otimes} \mathcal{A}\right)\right)^{*}\right)^{*}
$$

such that

$$
M . u=u . M, \quad u . \pi_{\sigma w c}(M)=u, u \in\left(\mathcal{M}_{m} \widehat{\otimes} \mathcal{A}\right) .
$$


Now as [24], we consider those elements of $\sigma w c\left(\left(\left(\mathcal{M}_{m} \widehat{\otimes} \mathcal{A}\right) \widehat{\otimes}\left(\mathcal{M}_{m} \widehat{\otimes} \mathcal{A}\right)\right)^{*}\right)^{*}$ that lies in the canonical image of $\left(\mathcal{M}_{m} \widehat{\otimes} \mathcal{A}\right) \widehat{\otimes}\left(\mathcal{M}_{m} \widehat{\otimes} \mathcal{A}\right)$ and we write $M=\sum_{i, j=1}^{m}\left(\xi_{i j} \otimes a_{i j}\right) \otimes \Sigma_{r, l=1}^{m}\left(\xi_{r l} \otimes b_{r l}\right)$.

Let $E$ be a normal Banach $\mathcal{A}$-bimodule with predual $E_{*}$ and $D: \mathcal{A} \longrightarrow E$ be a derivation that is weak ${ }^{*}$-continuous. By a similar argument in Lemma 3.3 in [8], we may assume that $E$ is a normal dual Banach $\mathcal{A}$-bimodule such that its predual is essential. Let

$$
\begin{aligned}
\psi:\left(\mathcal{M}_{m} \widehat{\otimes} \mathcal{A}\right) \widehat{\otimes}\left(\mathcal{M}_{m} \widehat{\otimes} \mathcal{A}\right) & \longrightarrow\left(\mathcal{M}_{m} \widehat{\otimes} \mathcal{M}_{m}\right) \widehat{\otimes}(\mathcal{A} \widehat{\otimes} \mathcal{A}) \\
\psi((c \otimes x) \otimes(d \otimes y)) & =(c \otimes d) \otimes(x \otimes y)\left(x, y \in \mathcal{A}, c, d \in \mathcal{M}_{m}\right)
\end{aligned}
$$

be the onto linear isometry. Let $c \in \mathcal{A}$ and $c=\Sigma_{s, t=1}^{m} \xi_{s t} \otimes c_{s t}$ that $c_{11}=c, c_{s t}=0$ if $s \neq 1$ or $t \neq 1$. We have

$$
\begin{aligned}
c=c . \pi_{\sigma w c}(M) & =\Sigma_{s, t=1}^{m} \xi_{s t} \otimes c_{s t} . \Sigma_{i, j, l=1}^{m}\left(\xi_{i l} \otimes a_{i j} b_{j l}\right) \\
& \left.=\sum_{i, j, s, l=1}^{m} \xi_{s l} \otimes c_{s i} a_{i j} b_{j l}\right) .
\end{aligned}
$$

Then

$$
\sum_{i, j, s, t=1}^{m} \xi_{s t} \otimes\left(c_{s t}-c_{s i} a_{i j} b_{j t}\right)=0
$$

Also

$$
\begin{aligned}
c . M & =\Sigma_{s, t=1}^{m}\left(\xi_{s t} \otimes c_{s t}\right) \cdot\left(\Sigma_{i, j=1}^{m}\left(\xi_{i j} \otimes a_{i j}\right) \otimes \Sigma_{r, l=1}^{m}\left(\xi_{r l} \otimes b_{r l}\right)\right) \\
& =\left(\Sigma_{i, j=1}^{m}\left(\xi_{i j} \otimes a_{i j}\right) \otimes \Sigma_{r, l=1}^{m}\left(\xi_{r l} \otimes b_{r l}\right)\right) . \Sigma_{s, t=1}^{m}\left(\xi_{s t} \otimes c_{s t}\right)=\text { M.c. }
\end{aligned}
$$

Therefore

$$
\sum_{s, t, r, l, j=1}^{m}\left(\xi_{s j} \otimes c_{s t} a_{t j}\right) \otimes\left(\xi_{r l} \otimes b_{r l}\right)=\sum_{i, j, r, l, t=1}^{m}\left(\xi_{i j} \otimes a_{i j}\right) \otimes\left(\xi_{r t} \otimes b_{r l} c_{l t}\right) .
$$

Apply $\psi$, we have

$$
\sum_{i, t, r, l, j=1}^{m}\left(\xi_{i j} \otimes \xi_{r l}\right) \otimes\left(c_{i t} a_{t j} \otimes b_{r l}\right)=\sum_{i, j, r, l, t=1}^{m}\left(\xi_{i j} \otimes \xi_{r t}\right) \otimes\left(a_{i j} \otimes b_{r l} c_{l t}\right) .
$$

Suppose that $c=\Sigma_{s, t=1}^{m} \xi_{s t} \otimes c_{s t}$ that $c_{11}=c, c_{s t}=0$ if $s \neq 1$ or $t \neq 1$. Then

$$
\Sigma_{r, j=1}^{m}\left(\xi_{1 j} \otimes \xi_{r 1}\right) \otimes\left(c_{11} a_{1 j} \otimes b_{r 1}\right)=\Sigma_{r, j=1}^{m}\left(\xi_{1 j} \otimes \xi_{r 1}\right) \otimes\left(a_{1 j} \otimes b_{r 1} c_{11}\right) .
$$

Define

$$
\begin{aligned}
\theta:\left(\left(\mathcal{M}_{m} \widehat{\otimes} \mathcal{M}_{m}\right) \widehat{\otimes}(\mathcal{A} \widehat{\otimes} \mathcal{A})\right) & \longrightarrow \\
\theta\left(\sum_{i, j, r, l=1}^{m}\left(\xi_{i j} \otimes \xi_{r l}\right) \otimes\left(a_{i j} \otimes b_{r l}\right)\right) & =\quad \sum_{i, j, r, l=1}^{m} a_{i j} D\left(b_{r l}\right) .
\end{aligned}
$$

It is easy to see that $\psi$ and $\theta$ are weak ${ }^{*}$-continuous. Now consider

$$
\lambda=\theta O \psi:\left(\mathcal{M}_{m} \widehat{\otimes} \mathcal{A}\right) \widehat{\otimes}\left(\mathcal{M}_{m} \widehat{\otimes} \mathcal{A}\right) \rightarrow E .
$$

From Lemma 4.9 in [24] $\lambda^{*}$ maps $E_{*}$ into $\sigma w c\left(\left(\left(\mathcal{M}_{m} \widehat{\otimes} \mathcal{A}\right) \widehat{\otimes}\left(\mathcal{M}_{m} \widehat{\otimes} \mathcal{A}\right)\right)^{*}\right)$ and so $\left(\left.\lambda^{*}\right|_{E_{*}}\right)^{*}$ maps $\sigma w c\left(\left(\left(\mathcal{M}_{m} \widehat{\otimes} \mathcal{A}\right) \widehat{\otimes}\left(\mathcal{M}_{m} \widehat{\otimes} \mathcal{A}\right)\right)^{*}\right)^{*}$ into $E$. We apply $\theta$ on (2.2) and we get

$$
\Sigma_{r, j=1}^{m} c_{11} a_{1 j} D\left(b_{r 1}\right)=\Sigma_{r, j=1}^{m} a_{1 j} D\left(b_{r 1} c_{11}\right) .
$$

Put $M_{1}=\Sigma_{r, j=1}^{m}\left(\xi_{1 j} \otimes a_{1 j}\right) \otimes\left(\xi_{r 1} \otimes b_{r 1}\right)$ and $M^{\prime}=\lambda\left(M_{1}\right)$. We obtain from (2.1) and (2.3),

$$
\begin{aligned}
\left\langle x, c . M^{\prime}\right\rangle & =\left\langle x, \Sigma_{r, j=1}^{m} c_{11} a_{1 j} D\left(b_{r 1}\right)\right\rangle \\
& =\left\langle x, \Sigma_{r, j=1}^{m} a_{1 j} D\left(b_{r 1} c_{11}\right)\right\rangle
\end{aligned}
$$




$$
\begin{aligned}
& =\left\langle x, \Sigma_{r, j=1}^{m} a_{1 j} D\left(b_{r 1}\right) \cdot c_{11}+\Sigma_{r, j=1}^{m} a_{1 j} b_{r 1} D\left(c_{11}\right)\right\rangle \\
& =\left\langle x, M^{\prime} . c\right\rangle+\langle x, D(c)\rangle
\end{aligned}
$$

for all $x \in E_{*}$. Consequently $D(c)=M^{\prime} \cdot c-c \cdot M^{\prime}$.

$(2) \Rightarrow(1)$ Let $E$ be a normal Banach $\mathcal{M}_{n} \widehat{\otimes} \mathcal{A}$-bimodule and $D: \mathcal{M}_{n} \widehat{\otimes} \mathcal{A} \longrightarrow E$ be a derivation that is weak*-continuous. Let $e_{\mathcal{A}}$ denote the identity of $\mathcal{A}$. We define

$$
\xi_{i j} \bullet x=\left(\xi_{i j} \otimes e_{\mathcal{A}}\right) . x, \quad x \bullet \xi_{i j}=x .\left(\xi_{i j} \otimes e_{\mathcal{A}}\right) \quad(i, j \in 1, . ., m) .
$$

So $E$ is a normal Banach $\mathcal{M}_{n}$-bimodule.

Put $D_{\mathcal{M}_{n}}: \mathcal{M}_{n} \longrightarrow E, D_{\mathcal{M}_{n}}\left(\xi_{i j}\right)=D\left(\xi_{i j} \otimes e_{\mathcal{A}}\right)$, then

$$
\begin{aligned}
D_{\mathcal{M}_{n}}\left(\xi_{i j} \xi_{k l}\right) & =D\left(\xi_{i j} \xi_{k l} \otimes e_{\mathcal{A}}\right) \\
& =D\left(\xi_{i j} \otimes e_{\mathcal{A}}\right) \cdot\left(\xi_{k l} \otimes e_{\mathcal{A}}\right)+\left(\xi_{i j} \otimes e_{\mathcal{A}}\right) . D\left(\xi_{k l} \otimes e_{\mathcal{A}}\right) \\
& =D\left(\xi_{i j} \otimes e_{\mathcal{A}}\right) \bullet \xi_{k l}+\xi_{i j} \bullet D\left(\xi_{k l} \otimes e_{\mathcal{A}}\right) \\
& =D_{\mathcal{M}_{n}}\left(\xi_{i j}\right) \bullet \xi_{k l}+\xi_{i j} \bullet D_{\mathcal{M}_{n}}\left(\xi_{k l}\right) .
\end{aligned}
$$

Hence, there exists $u \in E$ such that $D_{\mathcal{M}_{n}}=a d_{u}$. Therefore, $\tilde{D}=D\left(\xi_{i j} \otimes e_{\mathcal{A}}\right)-a d_{u}$ vanishes on $\mathcal{M}_{n} \otimes e_{\mathcal{A}}$.

Let $I$ be the identity matrix. Then $E$ is an $\mathcal{A}$-bimodule for the maps defined by

$$
a \circ x=(I \otimes a) . x, \quad x \circ a=x .(I \otimes a), \quad(a \in \mathcal{A}, \quad x \in E) .
$$

Let us now $D_{\mathcal{A}}(a)=\tilde{D}(I \otimes a)(a \in \mathcal{A})$. Define $K=\left\{e \in E_{*}:\langle\tilde{D}(I \otimes a), e\rangle=0\right\}$. Note that $\left(\frac{E_{*}}{K}\right)^{*}=\overline{\tilde{D}(I \otimes a)} w_{k}^{*}$. Further $\overline{\tilde{D}(I \otimes a)} w_{k}^{*}$ is a commutative normal Banach $\mathcal{A}$-bimodule. Then, there is $\nu \in \overline{\tilde{D}(I \otimes a)} w_{k}^{*}$ such that $\tilde{D}(I \otimes a)=(I \otimes a) . \nu-\nu .(I \otimes a)$. This complete the proof.

\section{Semigroup algebra}

In this section, we apply these results to semigroup algebra $l^{1}(S)$. For a semigroup $S$ and $s \in S$, we define maps $L_{s}, R_{s}: S \rightarrow S$ by $L_{s}(t)=s t, R_{s}(t)=t s, t \in S$. If for each $s \in S, R_{s}$ and $L_{s}$ are finite-to-one maps, then we say that $S$ is weakly cancellative. Before turning our results, we note that if $S$ is a weakly cancellative semigroup, then $l^{1}(S)$ is a dual Banach algebra with predual $c_{0}(S)$ [2]. It is known that $\left(l^{1}(S) \widehat{\otimes} l^{1}(S)\right)^{\prime}=B\left(l^{1}(S), l^{\infty}(S)\right)=l^{1}(S \times S)^{\prime}=l^{\infty}(S \times S)$, where $T \in B\left(l^{1}(S), l^{\infty}(S)\right)$ is identified with $T \in l^{\infty}(S \times S)$, where $T(s, t)=\left\langle T\left(\delta_{s}\right), \delta_{t}\right\rangle$. By the Krein-Smulian Theorem, $T$ is weakly compact if and only if the set $\left\{T\left(\delta_{s}\right): s \in S\right\}$ is relatively weakly compact.

A semigroup $S$ is simple if the only ideal in $S$ is $S$. A semigroup $S$ with zero is called 0 -simple if $\{0\}$ and $S$ are the only ideals and $S . S \neq 0$. An element $p \in S$ is an idempotent if $p^{2}=p$, the set of idempotents of $S$ is denoted by $E(S)$. For $p, q \in E(S)$, set $p \leq q$ if $p q=q p=p$. An element $e \in E(S)$ is called primitive if it is nonzero and is minimal in the set of nonzero idempotents. $\mathrm{S}$ is called completely simple if it is simple and contains a primitive idempotent.

Let $G$ be a group, $I$ and $J$ be arbitrary nonempty sets and $G^{0}=G \cup\{0\}$. Let $P_{G}=$ $\left(a_{i j}\right) \in M_{J \times I}(G)$. For $x \in G$, let $(x)_{i j}$ be the element of $M_{I \times J}\left(G^{0}\right)$ with $x$ in $(i, j)^{t h}$ place and 0 elsewhere. The set of all $(x)_{i j}$ matrices is denoted by $S$. Multiplication in $S$ is given by the formula

$$
(x)_{i j}(y)_{k l}=\left(x a_{j k} y\right)_{i l} \quad(x, y \in G, i, k \in I, j, l \in J) .
$$


We write $S=\mathcal{M}(G, P, I, J)$. $S$ is called Rees matrix semigroup with sandwich matrix $P$. It is known that $S$ is a completely simple semigroup and each completely simple semigroup is isomorphic to one constructed in this manner [10]. Similarly, we have the semigroup $\mathcal{M}^{0}(G, P, I, J)$ where the elements of this semigroup are those of $\mathcal{M}(G, P, I, J)$, together with the element 0 so that 0 is a matrix with 0 everywhere and $P_{G}=\left(a_{i j}\right) \in M_{J \times I}\left(G^{0}\right)$.

Proposition 3.1. Let $S$ be a weakly cancellative semigroup and let $l^{1}(S)$ be unital with unit $e_{l^{1}(S)}$. If $l^{1}(S)$ is Connes amenable, then $E(S)$ is finite.

Proof. For each $s \in S$, we put $\left[s s^{-1}\right]=\{x \in S: x s=s\}$ and $\left[s^{-1} s\right]=\{x \in S: s x=s\}$ and $\chi(s)=s S \cap\left[s s^{-1}\right]$. We follow [3] and consider the equivalence relation $R$ on $E(S)$ by $s R t$ if $s \in \chi(t)$. By this relation, $E(S)$ is partitioned into the sets $\chi(s)$. Suppose via contradiction, there exists an infinite sequence of sets $\chi\left(k_{n}\right),\left\{k_{n}\right\}_{n \in \mathbb{N}} \in S$.

Let $M \in\left(l^{1}(S) \widehat{\otimes} l^{1}(S)\right)^{* *}=l^{\infty}(S \times S)^{\prime}$ be a $\sigma w c$-virtual diagonal for $l^{1}(S)$ which satisfies Theorem 5.9 in [2]. Therefore $\langle M, f(h k, g)-f(h, k g)\rangle=0$ for each $k \in S$ and $f \in l^{\infty}(S \times S)$. Then $\left\langle M, f\left(h^{\prime}, g\right)\right\rangle=0,\left(h^{\prime}=h k, g \notin k S\right)$. In particular $\left\langle M, f\left(h^{\prime}, g\right)\right\rangle=0\left(h^{\prime}=h k, g \notin k S, g h k=k\right)$.

Now consider the multiplication map $\pi: l^{1}(S) \widehat{\otimes} l^{1}(S) \rightarrow l^{1}(S)$ and $f^{\prime} \in l^{\infty}(S)$ such that $\pi^{\prime} f^{\prime}=f$. We have $\left\langle\pi^{\prime}\left(f^{\prime}\right), \delta_{g} \otimes \delta_{h}\right\rangle=\left\langle f^{\prime}, \delta_{g h}\right\rangle$. Also

$$
\left\langle\delta_{k} \cdot M, \pi^{\prime}\left(f^{\prime}\right)\right\rangle=\left\langle\delta_{k} \cdot\left(e_{l^{1}(S)} \otimes e_{l^{1}(S)}\right), \pi^{\prime}\left(f^{\prime}\right)\right\rangle=\left\langle\left(\delta_{k} \cdot e_{l^{1}(S)} \otimes e_{l^{1}(S)}\right), \pi^{\prime}\left(f^{\prime}\right)\right\rangle .
$$

Consequently

$$
\begin{aligned}
\langle M, f(h k, g)\rangle & =\left\langle M, \pi^{\prime}\left(f^{\prime} . \delta_{k}\right)\right\rangle=\left\langle M,\left\langle f^{\prime}, \delta_{g h k}\right\rangle(g, h)\right\rangle \\
& =\left\langle f^{\prime} \delta_{k}, e_{\mathcal{A}}\right\rangle(g, h)(g h k=k) .
\end{aligned}
$$

This implies that $\left\langle M, f\left(h^{\prime}, g\right)\right\rangle=\left\langle f^{\prime} \delta_{k}, e_{\mathcal{A}}\right\rangle\left(g \in k S, g h^{\prime} k=k\right)$. Write $f(h, g)=\left\langle T\left(\delta_{h}\right), \delta_{g}\right\rangle$ where $T \in B\left(l^{1}(S), l^{\infty}(S)\right)$. Since $T$ is weakly compact. By Theorem 5.9 in [2], $\left\{T\left(\delta_{i}\right): i \in I\right\}$ is relatively weakly compact and so totally bounded [20]. Let

$$
Z(k)=\{(h, g) \in S \times S: g \in k S, g h k=k\} .
$$

Since $S$ is weakly cancellative, then each $\chi\left(k_{n}\right)$ is finite and also $Z\left(k_{n}\right)$ is contained in pairwise disjoint sets $\chi\left(k_{n}\right)$. Choose distinct elements $k_{1}, k_{2}, . ., k_{n} \in S$ with

$$
L=\min \left|\left\langle f^{\prime}, \delta_{k_{i}}\right\rangle\right|, \quad n L \geq\|M\| \sum_{i=1(h, g) \in Z\left(k_{i}\right)}^{n} \sup \left\{\left|\left\langle T\left(\delta_{h}\right), \delta_{g}\right\rangle\right|\right\} .
$$

Therefore

$$
\begin{aligned}
n L \leq \Sigma_{i=1}^{n}\left|\left\langle f^{\prime}, \delta_{k_{i}}\right\rangle\right| & =\sum_{i=1(h, g) \in Z\left(k_{i}\right)}^{n}|\langle M, f(h, g)\rangle| \\
& \leq\|M\| \Sigma_{i=1(h, g) \in Z\left(k_{i}\right)}^{n} \sup \left\{\left|\left\langle T\left(\delta_{h}\right), \delta_{g}\right\rangle\right|\right\} .
\end{aligned}
$$

This is a contradiction.

Example 1. Let $S$ be the natural numbers $\mathbb{N}$, with the product

$$
(m, n) \rightarrow m \vee n=\max \{m, n\} .
$$

$S$ is a semigroup with identity 1 and weakly cancellative. Clearly $E(S)=S$. Then $l^{1}(S)$ is a dual Banach algebra with predual $c_{0}(S)$ and $l^{1}(S)$ is not Connes amenable as $E(S)$ is infinite. 
It is easy to see that each weakly cancellative semigroup is simple. In fact, suppose that $\mathcal{I}$ be a left ideal of $S$ containing a nonzero element $i$, then

$$
S=S i \subseteq S \mathcal{I} \subseteq \mathcal{I}
$$

and so $\mathcal{I}=S$. Consequently, if $S$ is a weakly cancellative semigroup with $E(S)$ finite, then $S$ is completely simple semigroup and Rees matrix semigroup of the form $S=\mathcal{M}(G, P, I, J)$.

Theorem 3.1. Let $S$ be a weakly cancellative semigroup and let $l^{1}(S)$ be unital with unit $e_{l^{1}(S)}$. If $l^{1}(S)$ is Connes amenable, then $S$ is a Rees matrix semigroup of the form $S=\mathcal{M}(G, P, I, J)$, $\mathcal{L} M\left(l^{1}(G), P, I, J\right)$ has an identity and $l^{1}(G)$ is Connes amenable.

Proof. By Proposition 3.1, $S$ is a simple semigroup with $E(S)$ finite, then $S$ is a completely simple semigroup. Therefore, $S$ is a Rees matrix semigroup of the form $S=\mathcal{M}(G, P, I, J)$. By Proposition 5.6 in $[4], l^{1}(S)$ is isometrically algebra isomorphic to $\mathcal{L} M\left(l^{1}(G), P, I, J\right)$. Since $l^{1}(S)$ is Connes amenable, then $\mathcal{L} M\left(l^{1}(G), P, I, J\right)$ is Connes amenable and it has an identity. Also by Theorem 2.1, $l^{1}(G)$ is Connes amenable.

Theorem 3.2. Let $S$ be a weakly cancellative semigroup with $E(S)$ finite and let $l^{1}(S)$ be unital with unit $e_{l^{1}(S)}$. Then $S$ is a Rees matrix semigroup of the form $S=\mathcal{M}(G, P, I, J)$. With above notation, the following are equivalent:

(i) $l^{1}(S)$ is Connes amenable;

(ii) $\mathcal{L} M\left(l^{1}(G), P, I, J\right)$ has an identity and $l^{1}(G)$ is Connes amenable;

(iii) $l^{1}(S)$ is amenable.

Proof. $(i) \Rightarrow($ ii $)$ is Theorem 3.1 .

$($ ii $) \Rightarrow(i) \mathcal{L} M\left(l^{1}(G), P, I, J\right)$ has an identity, then $I$ and $J$ are finite and $P$ is invertible [4]. Since $l^{1}(G)$ is Connes amenable, then from Theorem 2.1, $\mathcal{L} M\left(l^{1}(G), P, I, J\right)$ is Connes amenable. By Proposition 5.6 in $[4], l^{1}(S)$ is isometrically algebra isomorphic to $\mathcal{L} M\left(l^{1}(G), P, I, J\right)$ and $l^{1}(S)$ is Connes amenable.

$($ ii $) \Leftrightarrow($ iii $)$ By Theorem 5.3 in $[22], l^{1}(G)$ is amenable if and only if $l^{1}(G)$ is Connes amenable. Then, this is Theorem 5.9 in [4].

Theorem 3.3. Let $S=\mathcal{M}(G, P, I, J)$ be a weakly cancellative semigroup. Let $l^{1}(S)$ be unital with unit $e_{l^{1}(S)}$. With above notation, the following are equivalent:

(i) $l^{1}(S)$ is Connes amenable;

(i) $l^{1}(S)$ is amenable.

Proof. This follows in the same manner as the proof of Theorem 3.2.

Example 2. Let $G$ be an amenable group. Let $J$ be finite of order $n$. Let $S=\mathcal{M}(G, P, 1, J)$ where $P$ is invertible. Let $(a)_{1 j}$ and $(b)_{1 l}$ be two non-zero elements of $S=\mathcal{M}(G, P, 1, J)$. It is easy to see that

$$
(a)_{1 j}\left(p_{j 1}{ }^{-1} a^{-1} b\right)_{1 l}=(b)_{1 l} .
$$

Then $S$ is a weakly cancellative semigroup. By [11], $l^{1}(G)$ is amenable. By Proposition 5.6 and Theorem 4.1 in $[4], l^{1}(S)$ is amenable. Also $l^{1}(G)$ is Connes amenable and by Theorem $2.1, l^{1}(S)$ is Connes amenable. 


\section{Acknowledgments}

I would like to thank the referee for his/her careful reading of our paper and many valuable suggestions.

\section{References}

[1] T. D. Blackmore, Weak amenability of discrete semigroup algebras, Semigroup Forum, $\mathbf{5 5}$ (1997), 196-205.

[2] M. Daws, Connes amenability of bidual and weighted semigroup algebras, Math. Scand., 99 (2006), 217-246.

[3] J. Duncan and A. L. T. Paterson, Amenability for discrete convolution semigroup algebras, Math. Scand., 66 (1990), 141-146.

[4] G. H. Esslamzadeh, Banach algebra structure and amenability of a class of matrix algebras with applications, J. Funct. Anal., 161 (1999), 364-383.

[5] G. H. Esslamzadeh, Representation theory and positive functionals of involutive $l^{1}$-Munn algebras, Semigroup Forum, 69 (2004), 51-62.

[6] G. H. Esslamzadeh, Duals and topological center of a class of matrix algebras with applications, Proc. Amer. Math. Soc., 128 (2000), 3493-3503.

[7] G. H. Esslamzadeh, B. Shojaee and A. Mahmoodi, Approximate Connes-amenability of dual Banach algebras, Bull. Belg. Math. Soc. Simon Stevin, 19 (2012), 193-213.

[8] A. Ghaffari and S. Javadi, $\varphi$-Connes amenability of dual Banach algebras, Bull. Iran. Math. Soc., 43 (2017), 25-39.

[9] A. Ya. Helemskii, Homological essence of amenability in the sense of $A$. Connes: the injectivity of the predual bimodule, Sb. Math., 68 (1991), 555-566.

[10] J. M. Howie, An Introduction to Semigroup Theory, Academic Press, San Diego, 1976.

[11] B. E. Johnson, Cohomology in Banach Algebras, Mem. Amer. Math. Soc., 127 (1972).

[12] B. E. Johnson, R. V. Kadison and J. Ringrose, Cohomology of operator algebras III, Bull. Soc. Math. France, 100 (1972), 73-79.

[13] A. T. Lau, Analysis on a class of Banach algebras with applications to harmonic analysis on locally compact groups and semigroups, Fund. Math., 118 (1983), 161-175.

[14] S. M. Maepa and O. T. Mewomo, On character amenability of semigroup algebras, Quaest. Math., 39 (2016), no . 3, 307-318.

[15] O. T. Mewomo and N. B. Okela, On approximate character amenability of Banach algebra, J. Nigerian Math. Soc., 32 (2013), 303-315.

[16] O. T. Mewomo and O. J. Ogunsola, On n-weak amenability of semigroup algebras, J. Nigerian Math. Soc., 32 (2013), 298-301. 
[17] O. T. Mewomo, Various notions of amenability in Banach algebras, Expo. Math., 29 (2011), no. 3, 283-299.

[18] W. D. Munn, On semigroup algebras, Math. Proc. Cambridge Philos. Soc., 51 (1955), 1-15.

[19] V. I. Paulsen, Completely Bounded Maps and Dilations, Pitman Res. Notes Math. Ser., 146 Longman, Harlow/New York, 1986.

[20] W. Rudin, Functional Analysis, McGraw Hill, New York, 1991.

[21] V. Runde, Amenability for dual Banach algebras, Studia Math., 148 (2001), 47-66.

[22] V. Runde, Connes amenability and normal virtual diagonals for measure algebras I, J. London Math. Soc., 67 (2003), 643-656.

[23] V. Runde, Connes amenability and normal virtual diagonals for measure algebras II, Bull. Austral. Math. Soc., 68 (2003), 325-328.

[24] V. Runde, Dual Banach algebras: Connes amenability, normal, virtual diagonals, and injectivity of the predual bimodule, Math. Scand., 95 (2004), 124-144.

Ali Ghaffari Department of Mathematics, University of Semnan, P. O. Box 35195-363, Semnan, Iran.

E-mail: aghaffari@semnan.ac.ir

Samaneh Javadi Faculty of Engineering- East Guilan, University of Guilan, P. O. Box 4489163157, Rudsar, Iran.

E-mail: s.javadi62@gmail.com

Ebrahim Tamimi Department of Mathematics, University of Semnan, P. O. Box 35195-363, Semnan, Iran.

E-mail: tamimi-ebrahim@semnan.ac.ir 